\title{
Identification of diverse innate lymphoid cells in human decidua
}

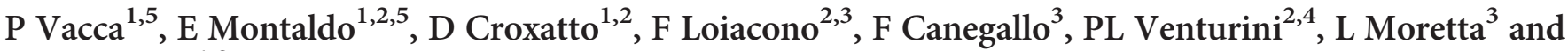 \\ MC Mingari ${ }^{1,2}$
}

Innate lymphoid cells (ILCs) are developmentally related cells that play an important role in innate defenses and tissue remodeling. So far, only natural killer (NK) cells have been identified and functionally characterized in human decidua where they contribute to induction of immune suppression, neo-angiogenesis, and tissue building/remodeling. The presence of other ILC subsets in human decidua has not been yet characterized. Here we identify in human decidua, during early pregnancy, two subsets of decidual group 3 ILC (ILC3), including lymphoid tissue inducer (LTi)-like cells and natural cytotoxicity receptors (NCRs) ${ }^{+}$ILC3 and interferon-(IFN) $\gamma$-producing ILC1, different from NK cells. Decidual LTi-like cells produced interleukin -17 (IL-17) and tumor necrosis factor (TNF), while NCR ${ }^{+}$ILC3 released IL-22 and IL-8. Importantly, $\mathrm{NCR}^{+}$ILC3 and LTi-like cells established functional interactions with stromal cells. Decidual LTi-like cells differentiated into $\mathrm{NCR}^{+}$ILC3, whereas they marginally contributed to NK cell generation. Our data suggest that decidual ILC3 may play a role in innate defenses and in vessel and tissue building, thus contributing to maintenance of pregnancy.

\section{INTRODUCTION}

Innate lymphoid cells (ILCs) are an extended family of developmentally related hematopoietic cells, which contribute to host immune defenses. Differentiation of ILC depends on the expression of the transcriptional repressor inhibitor of DNA binding 2 (ID2) and on the common cytokine receptor $\gamma$-chain. This shared requirement implies the existence of a common ILC progenitor. On the basis of their functional and developmental characteristics, three main groups of ILC have been identified: ILC1, ILC2, and ILC3. ${ }^{1,2}$ ILC1 include natural killer (NK) cells and other interferon (IFN) $\gamma$-producing cells. NK cells play a fundamental role in host defenses, thanks to their ability to kill virus-infected and tumor cells. In addition, they rapidly release cytokines and chemokines involved in early inflammatory responses., ${ }^{3,4}$ Human NK cell function is regulated by an array of inhibitory receptors, including killer Ig-like receptors (KIRs) and CD94/NKG2A, and activating receptors including NKp46, NKp30, and NKp44 (collectively known as natural cytotoxicity receptors (NCRs)), NKG2D,
DNAM-1, and CD16. ${ }^{5-9}$ During NK cell differentiation, other transcription factors (TFs) besides ID2 are required. ${ }^{10-14}$ While the nuclear factor interleukin (IL)-3-regulated (also known as E4BP4) TF is involved in early commitment to the NK cell lineage, the T-box expressed in $\mathrm{T}$ cells (Tbet/Tbx21) and Eomesodermin (Eomes) TFs are crucial for the acquisition of NK functional properties. ${ }^{15,16}$ Recently, other IFN $\gamma$-producing ILC1, different from NK cells, have been identified including non-cytotoxic $\mathrm{CD}_{2} 27^{+} \mathrm{CD} 117^{-} \mathrm{CD} 56^{-} \mathrm{CD} 94^{-}$ $\mathrm{NCR}^{-}$Tbet $^{+} \mathrm{IFN}^{+}{ }^{+}$cells ${ }^{17}$ and intraepithelial $\mathrm{CD} 127^{-}$ $\mathrm{CD}_{56}{ }^{+} \mathrm{NKp} 44^{+} \mathrm{CD} 103^{+}$ILC1. $^{18}$ ILC2 require GATA-binding protein 3 and RAR-related orphan receptor $\alpha$ TFs for their development. They are characterized by CD127, CD161, and chemoattractant receptor-homologous molecule expressed on $\mathrm{T}$ helper 2 cells (CRTH2) expression, and produce $\mathrm{T}$ helper 2-associated cytokines. ${ }^{19}$ Finally, group 3 ILC includes different cell types, defined by the requirement of ROR $\gamma \mathrm{t}$ TF for their development and function and by their ability to produce IL-17 and/or IL-22. ${ }^{2,20}$ Lymphoid tissue inducer (LTi) cells, the first

\footnotetext{
${ }^{1}$ Dipartimento di Medicina Sperimentale, Università di Genova, Genova, Italy. ${ }^{2}$ RCCS AOU San Martino-IST, Genova, Italy. ${ }^{3}$ Istituto Giannina Gaslini, Genova, Italy and ${ }^{4}$ Dipartimento di Neuroscienze, riabilitazione, oftalmologia, genetica e scienze materno-infantili, Università di Genova, Genova, Italy. Correspondence: $L$ Moretta (lorenzomoretta@ospedale-gaslini.ge.it)

${ }^{5}$ The first two authors contributed equally to this work.
} 
described ILC3, play a key role in the formation of secondary lymphoid organs (SLO) during fetus development. ${ }^{20}$ Notably, cells with similar phenotypic characteristics were identified also in adult SLO and were named LTi-like cells. In humans, LTi/ LTi-like cells express CD127 and CD117.,21 A third cell population belonging to group 3 ILC, termed $\mathrm{NK} 22$ or $\mathrm{NCR}^{+}$ ILC3, has been recently identified in mucosal tissues. The $\mathrm{NCR}^{+}$ILC3 population shares common features with NK cells and ILC3, as they express NCRs (NKp44 ${ }^{\text {high }} \mathrm{NKp} 46^{\text {low }}$ ), ROR $\gamma \mathrm{t}$ $\mathrm{TF}$, and produce IL-22. ${ }^{22-25}$

Regarding the possible role of ILC during pregnancy, so far only NK cells have been identified and functionally characterized in human decidua, where they are thought to contribute to the establishment of an immunosuppressive environment and to play a role in tissue building. ${ }^{26,27}$ Notably, a successful pregnancy requires an early inflammatory phase necessary for implantation, while later the establishment of a regulatory/ immunosuppressive phase is needed to prevent fetal rejection. ${ }^{28}$ During the first trimester, decidual NK (dNK) cells represent as much as $50-70 \%$ of decidua-infiltrating lymphocytes (DILs). ${ }^{29}$ Previous studies indicated that dNK cells may derive from $\mathrm{CD} 34^{+}$hematopoietic precursors present in situ (dCD34) and/or from immature NK cells already present in endometrial tissue, and/or from peripheral blood NK cells migrated to decidua during the early phase of pregnancy. ${ }^{30-32}$ dNK cells display unique phenotypic characteristics, are poorly cytolytic, ${ }^{33,34}$ and, unlike peripheral blood NK cells, release cytokines/chemokines involved in neo-angiogenesis, tissue remodeling, and placentation. ${ }^{26,29,35,36}$ In addition, dNK cells have been shown to induce regulatory $\mathrm{T}$ (Tregs) cells in vitro, i.e., cells that are thought to play a major role in the inhibition of maternal immune response and in tolerance induction. ${ }^{37}$

In the present study, we identified two additional decidual ILC3 populations, namely LTi-like cells and $\mathrm{NCR}^{+}$ILC3 and an ILC1 subset that differs from NK cells. Decidual LTi-like cells produced IL-17 and tumor necrosis factor (TNF), while $\mathrm{NCR}^{+}$ILC3 produced IL-22 and IL-8, whereas decidual ILC1 primarily released IFN- $\gamma$. LTi-like cells underwent in vitro differentiation into $\mathrm{NCR}^{+}$ILC3, whereas both $\mathrm{NCR}^{+} \mathrm{ILC} 3$ and LTi-like cells only marginally contributed to NK cell generation. Our data suggest that not only dNK cells, but also decidual ILC3 and ILC1 may play a relevant role both in innate defenses and in tissue building/remodeling during the early phases of pregnancy.

\section{RESULTS}

\section{Human decidua contains different ILC subsets}

Different ILC subsets are present in different organs and are characterized by the secretion of cytokines such as IL-17A, IL-22, IFN- $\gamma$, and TNF that promote/mediate tissues remodeling, lymphoid organogenesis, inflammation, and antimicrobial immunity. ${ }^{2}$ We investigated whether cells producing these cytokines were present in human decidua. To this end, DILs were stimulated with phorbol 12-myristate 13-acetate (PMA)/ ionomicyn/IL-23. After $18 \mathrm{~h}$, the cytokines produced by different cell subsets were analyzed by using the gating strategy shown in Figure 1a. Lineage negative $\left(\mathrm{Lin}^{-}\right)$cells $\left(\mathrm{CD} 45^{+}\right.$ $\mathrm{CD} 34^{-} \mathrm{CD} 19^{-} \mathrm{CD} 14^{-} \mathrm{CD} 3^{-}$) were gated as $\mathrm{CD} 56^{+} \mathrm{CD} 94^{+}$ (bona fide $\mathrm{NK}$ cells), $\mathrm{CD} 56^{+} \mathrm{CD} 94^{-}$, or $\mathrm{CD} 56^{-} \mathrm{CD} 94^{-}$. As shown in Figure 1b,d, $\mathrm{CD} 56^{+} \mathrm{CD} 94^{+} \mathrm{NK}$ cells produced primarily IFN- $\gamma$. CD56 ${ }^{+} \mathrm{CD} 94^{-}$cells included IFN $\gamma$-positive cells and cells that were IFN $\gamma$-negative but produced IL-22 and IL-8. In addition, a small fraction of $\mathrm{CD} 56^{+} \mathrm{CD} 94^{-}$cells produced IL-17A and TNF. The CD56 ${ }^{-} \mathrm{CD} 94^{-}$cell population express IL-17A and TNF, but not IL-22. None of the three cell subsets produced IL-2 (Figure 1b). Previous studies revealed that dNK cells, identified as $\mathrm{CD}^{-}{ }^{-} \mathrm{CD} 56^{+}$cells, released high amounts of IL-8, a chemokine playing an important role in pregnancy. ${ }^{26,35}$ Our present results indicate that high percentages of $\mathrm{IL}-8^{+}$cells belong to a $\mathrm{CD} 56^{+} \mathrm{CD} 94^{-}$cell subset, rather than to $\mathrm{CD} 56^{+} \mathrm{CD} 94^{+} \mathrm{NK}$ cells. Moreover, decidual $\mathrm{CD} 56^{+} \mathrm{CD} 94^{-} \mathrm{IL}-8^{+}$cells also produced IL-22, similar to ILC3 identified in tonsils. ${ }^{38,39}$ Conversely, CD $56^{+} \mathrm{CD} 94^{-}$ IFN $\gamma$-producing cells may represent ILC1 distinct from "conventional NK" cells. ${ }^{18}$ Our results also suggest that decidual tissue may contain ILC1 and ILC3 also among $\mathrm{CD}^{-} 6^{-}$cell population. Indeed, we identified $\mathrm{CD} 56^{-}$cells expressing IL-17A and TNF, putative LTi-like cells, and IFN- $\gamma^{+}$cells that may represent ILC1 ${ }^{17}$ (Figure 1b-d). Therefore, in order to verify whether cell populations identified in human decidua indeed included ILC3 and ILC1, a more detailed phenotypic characterization was required.

\section{Characterization of decidual ILC3 and ILC1}

The putative decidual ILCs were further analyzed for the expression of CD127, CD117, and NKp44, i.e., markers allowing a more precise identification of ILC subsets. $\mathrm{CD} 56^{+}$ CD $94^{+}$NK cells were negative for CD127 and CD117 and, when sorted, they produced only IFN- $\gamma$ and TNF (Figure 2a and Supplementary Figure S1a online). CD127 and CD117 were expressed only by a fraction of $\mathrm{CD} 56{ }^{+} \mathrm{CD} 94^{-}$cells that homogenously expressed also $\mathrm{NKp} 44$, as previously described for $\mathrm{NCR}^{+}{ }^{\mathrm{ILC}} 3 .^{1,2}$ When sorted, $\mathrm{CD} 56^{+} \mathrm{CD} 94^{-} \mathrm{CD} 127^{+}$ $\mathrm{CD}_{117^{+}}$cells produced high amount of IL-8 and IL-22, while they were negative for IFN- $\gamma$ (Supplementary Figure S1a). Thus, $\mathrm{CD} 56^{+} \mathrm{CD} 94^{-} \mathrm{CD} 127^{+} \mathrm{CD} 117^{+} \mathrm{NKp} 44^{+}$cells represent decidual $\mathrm{NCR}^{+}$group 3 ILC and are hereinafter referred to as $\mathrm{NCR}^{+}$ILC3. Moreover, among $\mathrm{Lin}^{-} \mathrm{CD} 56^{-}$ cells, we identified a subset of $\mathrm{CD} 127^{+} \mathrm{CD} 117^{+}$cells that produced IL-17A and TNF, similarly to LTi-like cells previously described $^{1,2}$ (Figure 2a and Supplementary Figure S1a). Thus, $\mathrm{Lin}^{-} \mathrm{CD} 56^{-} \mathrm{CD} 127^{+} \mathrm{CD} 117^{+}$cells likely represent decidual LTi-like cells, and are hereinafter referred to as LTi-like cells (Figure 2a). Moreover, as shown above (Figure 1b), we observed that IFN- $\gamma$ expression was not restricted to $\mathrm{CD} 56^{+} \mathrm{CD} 94^{+} \mathrm{NK}$ cells. Indeed, also $\mathrm{CD} 56^{+} \mathrm{CD} 94^{-}$ $\left(\mathrm{CD} 127^{-}\right) \mathrm{CD}_{117^{-}}$cells produced IFN- $\gamma$ (Supplementary Figure S1b). These cells expressed Tbet and Eomes, but not ROR $\gamma$ t TF. Notably, similarly to intraepithelial ILC1 (iILC1) identified in gut, ${ }^{18}$ they were $\mathrm{CD} 103^{+} \mathrm{NKp} 44^{-/ \text {low }}$. Interestingly, in decidual $\mathrm{CD}^{-} \mathrm{CD}^{-} 6^{-}\left(\mathrm{CD} 127^{-}\right) \mathrm{CD}_{117^{-}}$subset, 


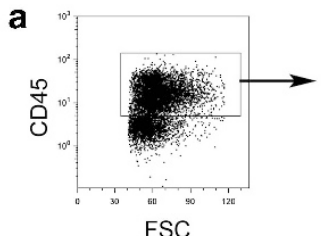

b

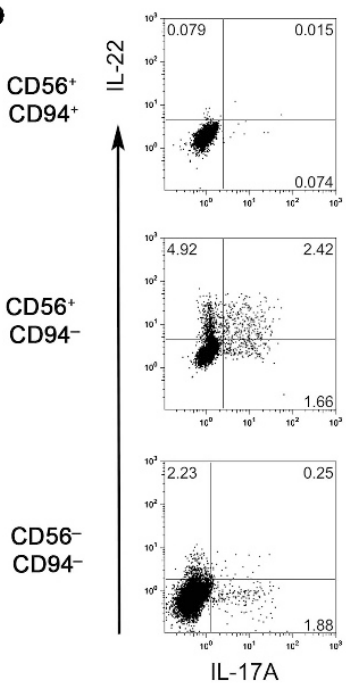

FSC

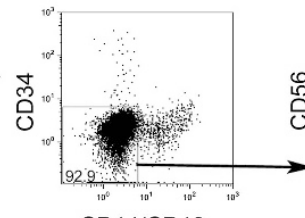

CD14/CD19

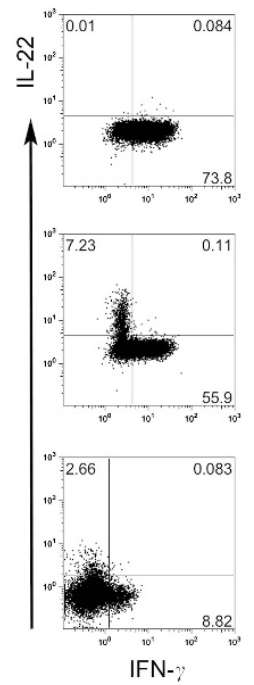

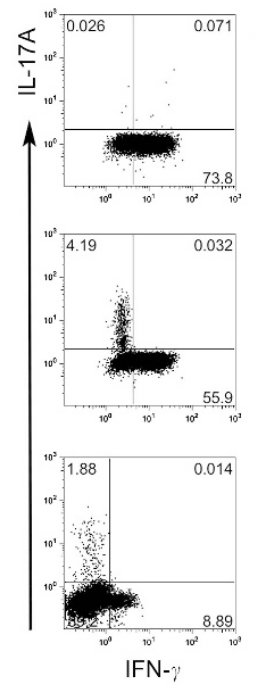

IFN- $\gamma$

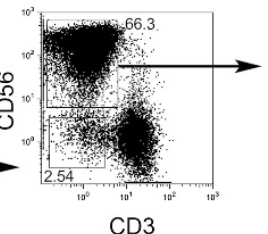

CD3

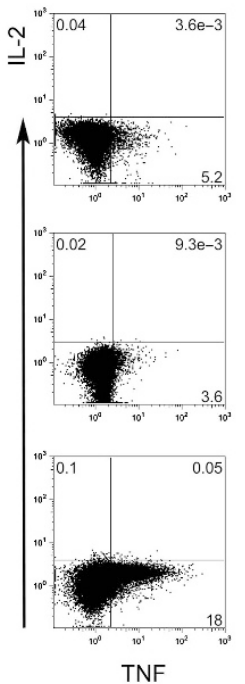

TNF

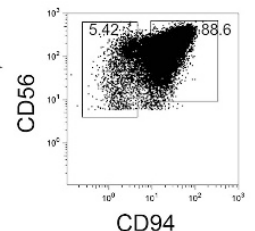

CD94

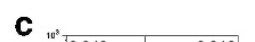

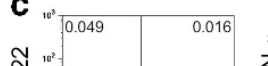

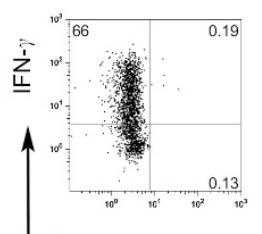

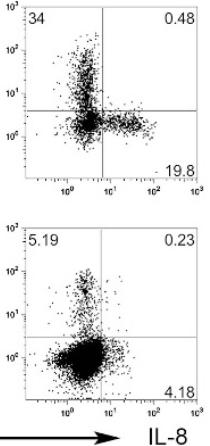
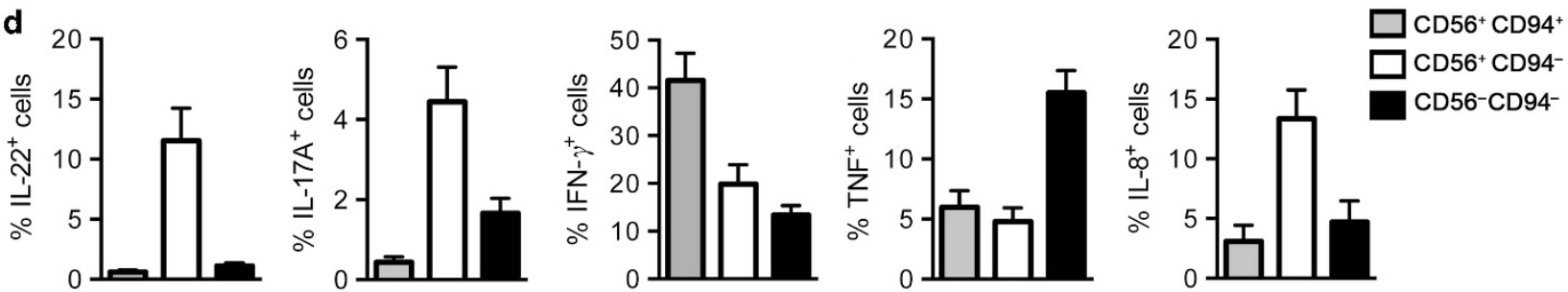

Figure 1 Cytokine profile of decidual ILCs. Decidua-infiltrating lymphocytes (DILs) were stimulated ex vivo and analyzed by flow cytometry for the expression of the specified markers and cytokines. (a) Gating strategy according to surface markers expression. (b-d) Intracellular analysis of the indicated cytokines, after 18-h stimulation with PMA/ionomicyn/IL-23. We identified three different cell populations according to the expression

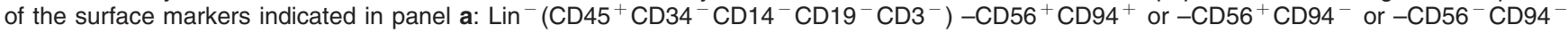
cells. (b, c) One representative experiment out of six performed. (d) Mean \pm s.e.m. of cytokine-positive cells.

we could also identify IFN- $\gamma^{+}$Tbet $^{+}$Eomes ${ }^{-}$ROR $\gamma \mathrm{t}^{-}$cells, similar to ILC1 described by Bernink et al. (Supplementary Figure S1b). ${ }^{17}$ These data indicate that ILC1 different from NK cells are present in human decidua.

A fundamental requirement to define both $\mathrm{NCR}^{+}$ILC3 and LTi-like cells is the expression of ROR $\gamma \mathrm{t}$ TF. As shown in Figure $2 \mathbf{b}, \mathbf{c}$, unlike NK cells, decidual $\mathrm{NCR}^{+}$ILC3 and LTi-like cells expressed ROR $\gamma$ t. On the contrary, NK cells expressed the Eomes TF. In order to further characterize the subset assignment of decidual ILC, we next assessed different markers associated with NK cells, $\mathrm{NCR}^{+}$ILC3, or LTi-like cells. As shown in Figure 2d, both $\mathrm{NCR}^{+}$ILC3 and LTi-like cells expressed higher levels of IL-23R, IL-1R1, CCR6, and RANKL compared with NK cells. In line with previous reports, decidual LTi-like cells expressed higher levels of CD25 as compared with NK cells and $\mathrm{NCR}^{+}$ILC3, while all cell subsets were CD62L negative and displayed bimodal expression of
CD7. ${ }^{21,24}$ In addition, NCR ${ }^{+}$ILC3 displayed lower LFA-1 expression them NK and LTi-like cell subsets. ${ }^{40}$ On the contrary, $\mathrm{NCR}^{+}$ILC3 expressed higher levels of CXCR5, CD161, and NKp44 compared with NK cells and LTi-like cells. Conversely, NK cells expressed much higher levels of NKp46, NKG2D, KIRs, perforin, and granzyme B compared with $\mathrm{NCR}^{+}$ILC3 and LTi-like cells. Finally, CRTH 2 was barely detectable in all subsets, which suggested that decidual ILC may not contain ILC2. ${ }^{19}$ Taken together, these data further confirm that both $\mathrm{NCR}^{+}$ILC3 and LTi-like cells are present in human decidua during the first trimester of pregnancy.

\section{Decidual NCR ${ }^{+}$ILC3 and LTi-like cells respond to cytokine stimulation}

It has been shown that upon stimulation with a combination of IL-7, IL-1 $\beta$, and IL-23, ILC3 release cytokines including IL-22. ${ }^{38,41}$ As shown in Figure 3a,b, decidual $\mathrm{NCR}^{+}$ILC3 
a

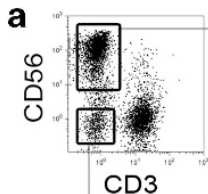

CD3

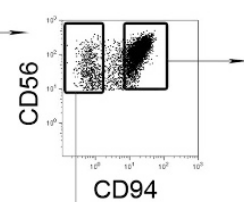

CD94

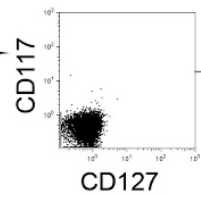

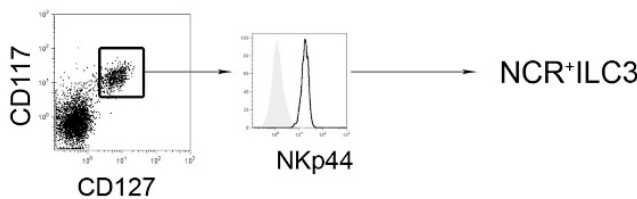

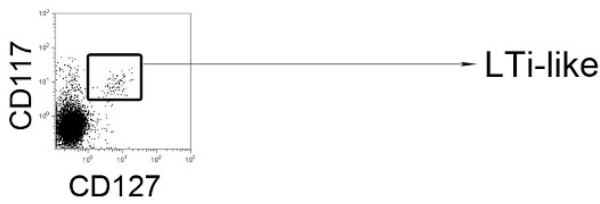

- NK

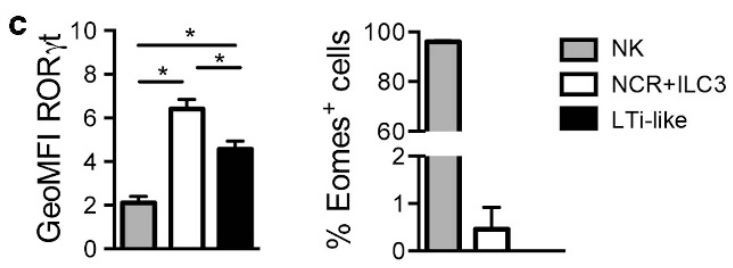

b

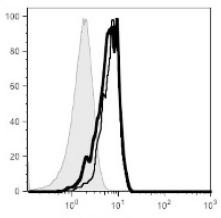

ROR $\gamma \mathrm{t}$

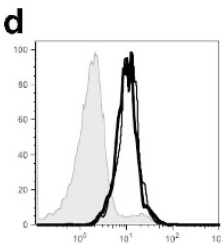

IL-23R

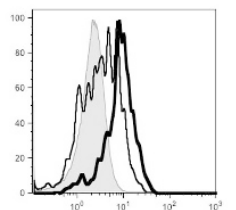

CD25

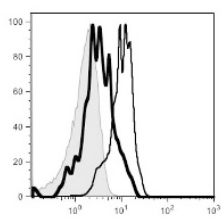

NKp44

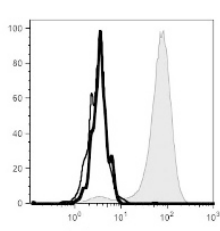

Eomes

NK

- NCR ${ }^{+}$ILC3

— LTi-like

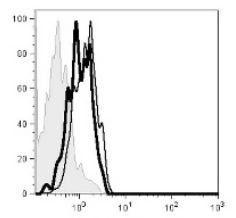

IL-1R1

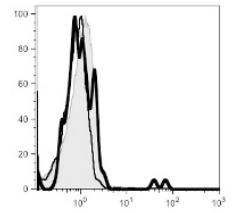

CD62L

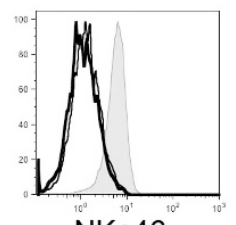

NKp46

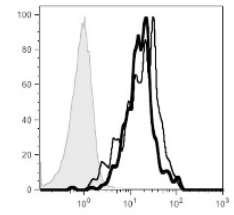

CCR6

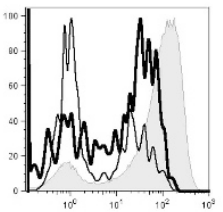

CD7

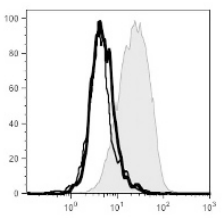

NKG2D

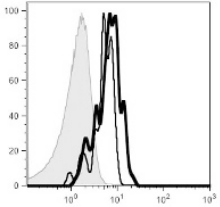

RANKL

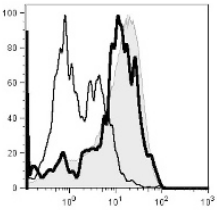

LFA-1

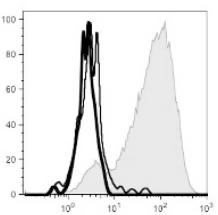

KIRs

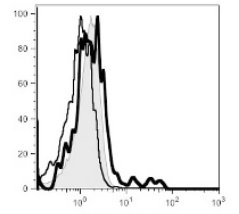

CRTH2

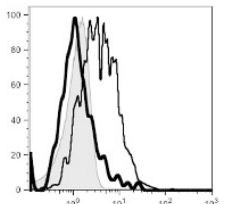

CXCR5

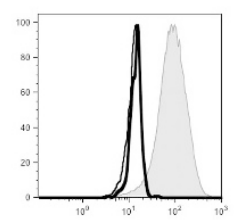

Perforin
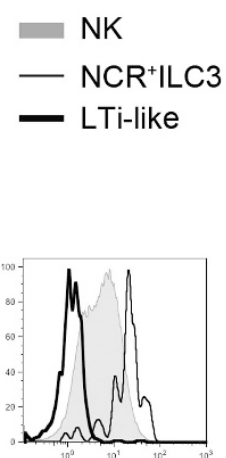

CD161

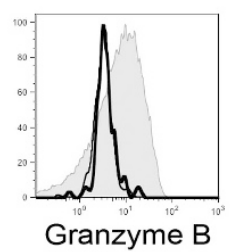

Figure 2 Phenotypic analysis of decidual ILCs. DILs were analyzed ex vivo by flow cytometry for the expression of the specified markers. (a) Identification of different ILC subsets by surface marker analysis. (b-c) Intranuclear expression of the indicated transcription factors

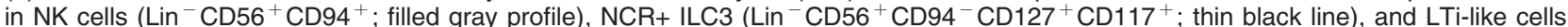
$\left(\mathrm{Lin}^{-} \mathrm{CD} 56^{-} \mathrm{CD} 127^{+} \mathrm{CD} 117^{+}\right.$; thick black line). (b) One representative experiment out of seven performed. (c) Mean \pm s.e.m. of ROR $\gamma \mathrm{t}$ geoMFI and percentage of Eomes-positive cells. ${ }^{*}=P \leq 0.05$. (d) Surface expression of the indicated markers in NK cells (filled gray profile), NCR ${ }^{+}$ILC3 (thin black line), and LTi-like cells (thick black line). One representative experiment out of five performed.

produced IL-22 in response to IL-7/IL-1 $\beta /$ IL-23 stimulation. In addition, NK cells and LTi-like cells did not produce IL-22 in response to IL-7/IL-1 $\beta /$ IL-23 stimulation (not shown). Upon interaction with stromal cells, ILC3 have been shown to induce the expression of ICAM1 and VCAM1 adhesion molecules. ${ }^{21,24}$ Thus, we investigated whether decidual $\mathrm{NCR}^{+}$ILC3 and LTi- 
a

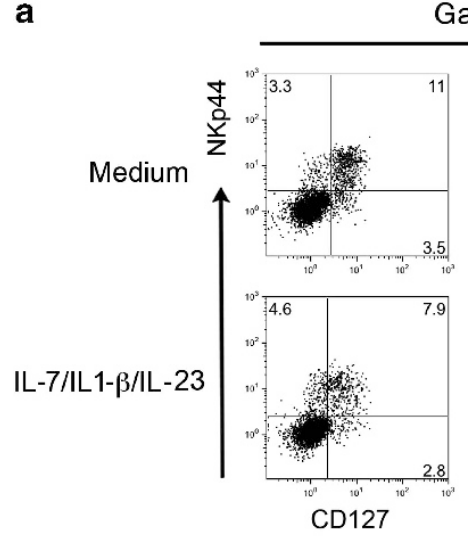

Gated on Lin-CD56+CD94-

C
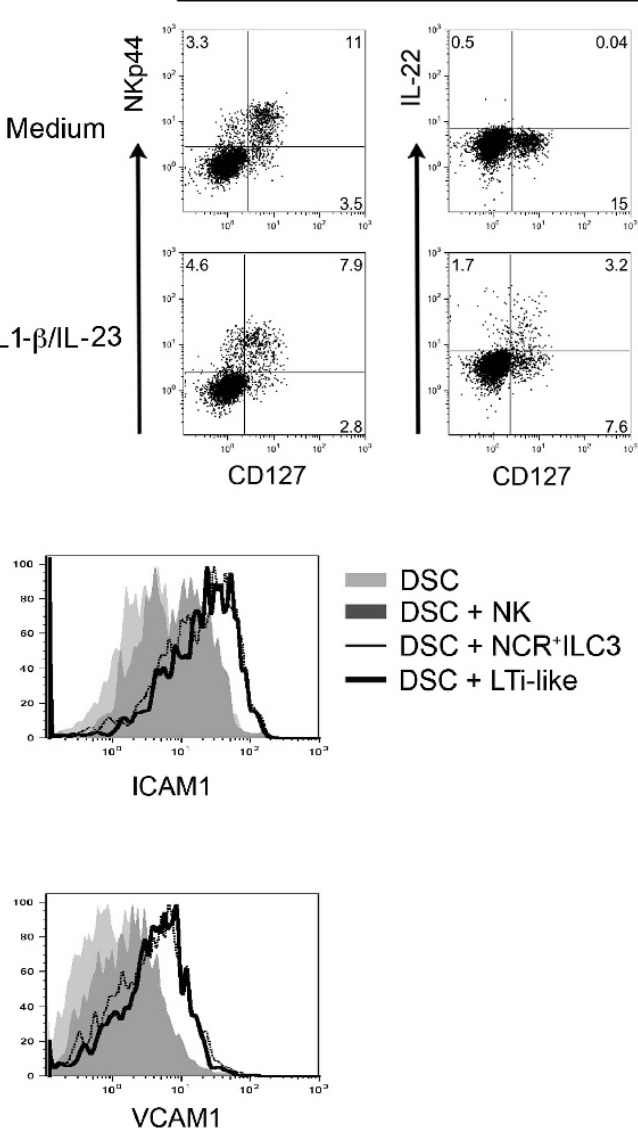

b

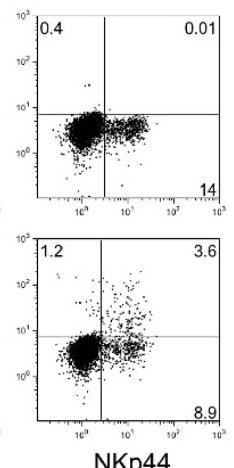

$\operatorname{Lin}^{-}{ }^{-C D} 56^{+}{ }^{-C D} 94^{-C D} 127^{+}$
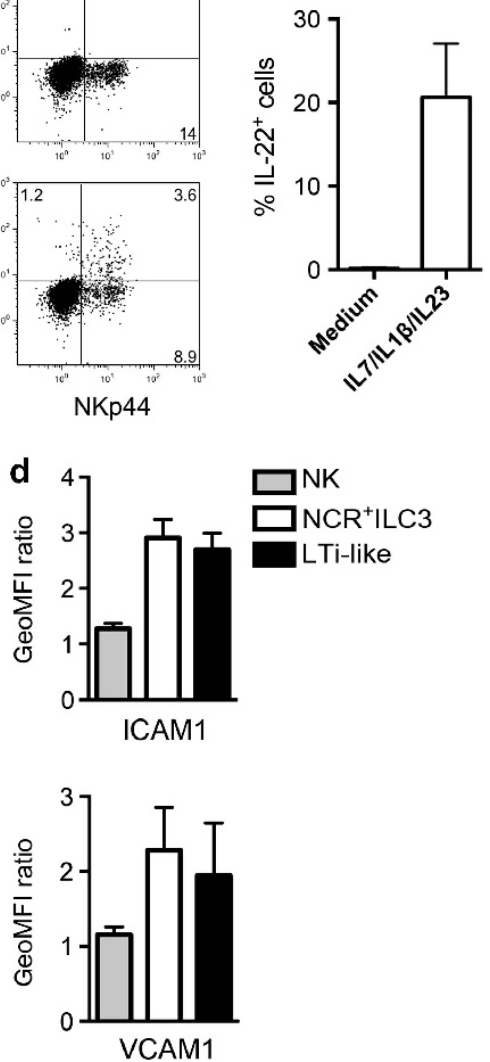

Figure 3 Decidual ILC3 produce IL-22 in response to cytokine stimulation and induce upregulation of adhesion molecules on decidual stromal cell (DSC). (a, b) DILs were left untreated (medium) or stimulated with IL-7/IL-1//IL-23 for $18 \mathrm{~h}$ and analyzed for IL-22 production and CD127 and NKp44 expression after gating on $\mathrm{Lin}^{-} \mathrm{CD}^{-} 6^{+} \mathrm{CD}^{-} 4^{-}$cells. (a) One representative experiment out of three performed. (b) Mean \pm s.e.m. of IL-22-positive cells; cells were gated on $\mathrm{Lin}^{-} \mathrm{CD} 56^{+} \mathrm{CD} 94^{-} \mathrm{CD} 127^{+}$cells. (c, d) DSC were cultured for 7 days in IL-7 in the presence or in the absence of sorted NK cells

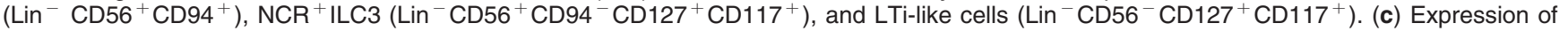
ICAM1 and VCAM1 on DSC was analyzed by flow cytometry. One representative experiment out of three was performed. (d) ICAM1 and VCAM1 expression, calculated as ratio of geoMFI of DSC + cells to DSC alone. Mean \pm s.e.m. of three independent experiments.

like cells could induce upregulation of these adhesion molecules on decidual stromal cells (DSCs). Sorted NK cells, NCR ${ }^{+}$ILC3, and LTi-like cells were cocultured with DSCs. After 7 days, DSCs displayed higher expression of ICAM1 and VCAM1 when cultured with $\mathrm{NCR}^{+}$ILC3 and LTi-like cells as compared with DSCs cultured with NK cells (Figure 3c,d). These data reveal that cells with LTi activity are present in human decidua.

\section{ILC3 proliferate and partially differentiate toward NK cells}

We analyzed whether decidual ILC3 could undergo in vitro proliferation in the presence of cytokines previously shown to promote survival and proliferation of ILC3 isolated from tonsil. $^{21,41}$ The decidual $\mathrm{NCR}^{+}$ILC3 and LTi-like cells represent a small fraction of total $\mathrm{CD} 45^{+}$cells. However, their numbers $/ 10^{6} \mathrm{CD} 45^{+}$cells in decidua were comparable with, if not higher, than that of tonsil ILC3 subsets (Supplementary Figure S2a). In the presence of IL-2 and IL-7, we observed only marginal proliferation of decidual
$\mathrm{NCR}^{+}$ILC3. Moreover, the combined use of IL-7/IL-2 and IL-7/IL-1 $\beta$ could slightly increase cell proliferation. Much higher proliferation occurred in the presence of feeder cells (irradiated allogeneic peripheral blood mononuclear cells + LCL $221^{\star} \mathrm{G}$ cells) and IL-2 or IL-15 (Supplementary Figure S2b). Notably, NCR ${ }^{+}$ILC3 and LTi-like cells displayed a much higher proliferative capacity in comparison with NK cells (Supplementary Figure S2c).

IL-15-expanded NK cells maintained their phenotype and upregulated NKp44 expression, compared with freshly isolated cells (Figure 4a,b). As previously described, CD127 was no longer detectable after culture on both $\mathrm{NCR}^{+} \mathrm{ILC}_{3}$ and LTilike cells (Figure 4a). ${ }^{21}$ Cultured $\mathrm{NCR}^{+}$ILC3 expressed CD117, CCR6, RANKL, NKp44 and upregulated NKp46 (Figure 4a,b). Interestingly, a consistent fraction of cultured LTi-like cells seemed to differentiate toward $\mathrm{NCR}^{+}$ILC3 as suggested by the acquisition of CD 56 and NKp44 (Figure 4a,b). In addition, a fraction of cultured $\mathrm{NCR}^{+}$ILC3 and CD56 ${ }^{+}$ 

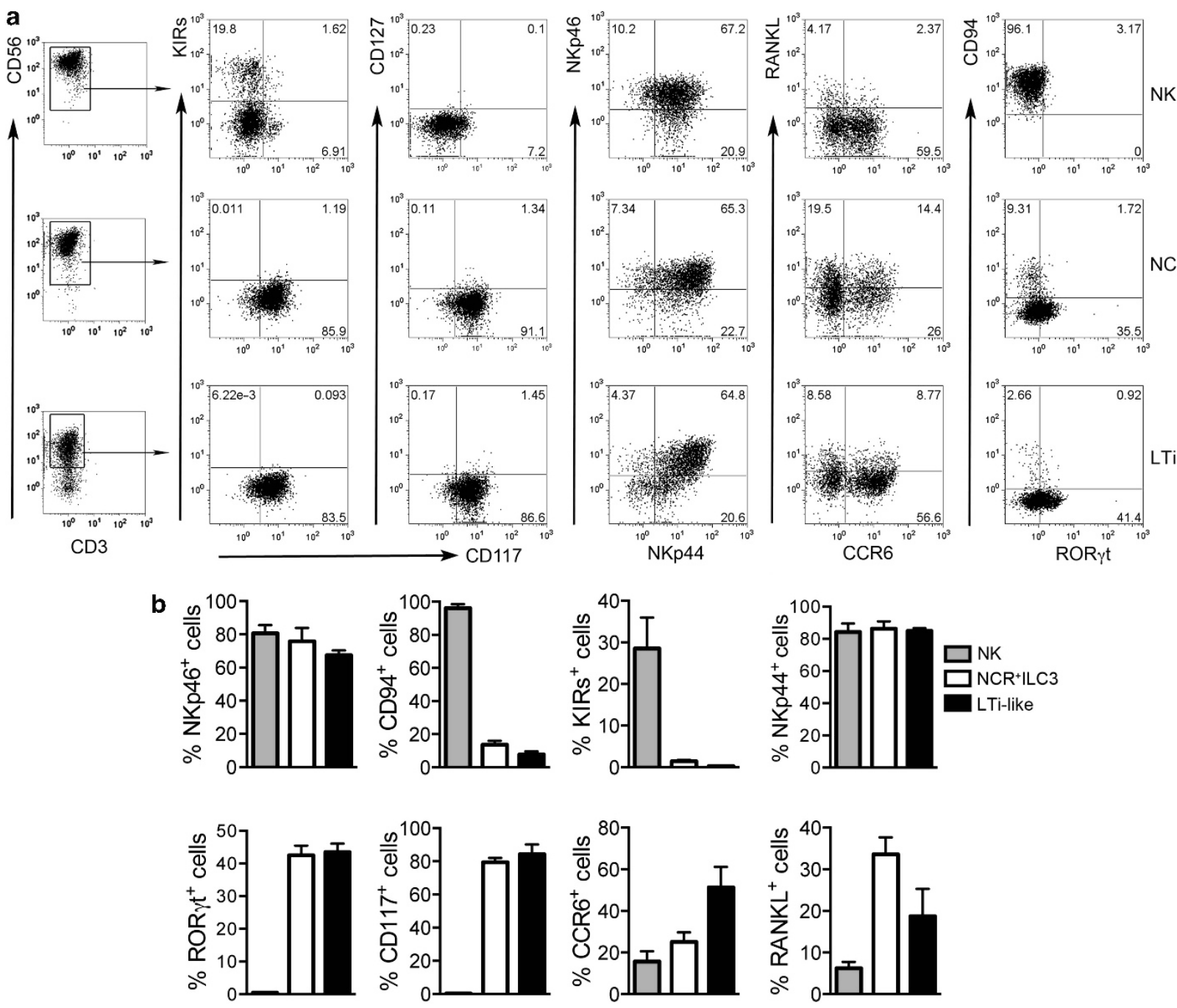

Figure 4 Phenotypic analysis of decidual ILCs after culture. NK cells, NCR ${ }^{+}$ILC3, and LTi- like cells were sorted from decidua and cultured for 20 days in the presence of IL-15. Phenotypic analysis of indicated markers after gating on CD56 ${ }^{+}$cells. (a) One representative experiment out of eight was performed. (b) Mean \pm s.e.m. of percentage of positive cells.

LTi-like cells acquired CD94, a typical feature of NK cells (Figure 4a,b). However, $\mathrm{NCR}^{+} \mathrm{ILC}_{3}$ and $\mathrm{CD} 56^{+}$LTi-like cells expressed low levels of LFA-1 (acquired during NK cell differentiation ${ }^{39,40}$ ) and were KIR negative (Figure $\mathbf{4 a , b}$ and Supplementary Figure S2d). These results suggest that LTilike cells could in part differentiate toward $\mathrm{NCR}^{+} \mathrm{ILC} 3$ and that LTi-like cells and $\mathrm{NCR}^{+}$ILC3 could partially differentiate toward $\mathrm{CD} 6^{+} \mathrm{CD} 117^{+} \mathrm{CD} 94^{+} \mathrm{LFA}_{-1} 1^{\text {low }} \mathrm{KIR}^{-}$cells that might represent immature NK cells. ${ }^{42}$ Similar results were obtained in cultures with IL-2 (Supplementary Figure S2e-f). In order to verify the differentiation potential of $\mathrm{NCR}^{+}$ILC3 and LTi-like cells, limiting dilution experiments were performed, in comparison with $\mathrm{NK}$ cells. As shown in Supplementary Figure S3a, NCR ${ }^{+}$ILC3 and LTi-like cells displayed a higher cloning efficiency than that of NK cells. All NK clones maintained their initial surface phenotype, thus, they expressed CD94 and remained CD117 negative
(Supplementary Figure S3b,d). In line with the results obtained in bulk cultures (see Figure $4 \mathbf{a}, \mathbf{b}$ ), a fraction of LTi-like clones acquired CD56 and NKp44, indicating that they can undergo differentiation toward $\mathrm{NCR}^{+} \mathrm{ILC} 3$ (Supplementary Figure S3c and data not shown). In addition, some $\mathrm{NCR}^{+} \mathrm{ILC} 3$ and $\mathrm{CD} 6^{+}$LTi-like clones partially acquired CD94, but not KIRs. The presence of CD94 ${ }^{+}$cells in ILC3 cultures might be due to NK cell contamination. However, similarly to freshly isolated ILC3, the CD $94^{+}$cells obtained from ILC3 cultures expressed CD117 (Supplementary Figure S3b-d and data not shown). Fresh and cultured NK cells did not express CD117 and displayed lower cloning efficiency than $\mathrm{NCR}^{+}$ILC3 and LTi-like cells. All these findings suggest that, upon culture with cytokines, decidual ILC3 can give rise to $\mathrm{CD} 117^{+} \mathrm{CD} 94^{+}$cells that may represent putative immature NK cells. ${ }^{42,43}$ Notably, some ILC3-derived clones maintained their initial phenotype and did not acquire 
a
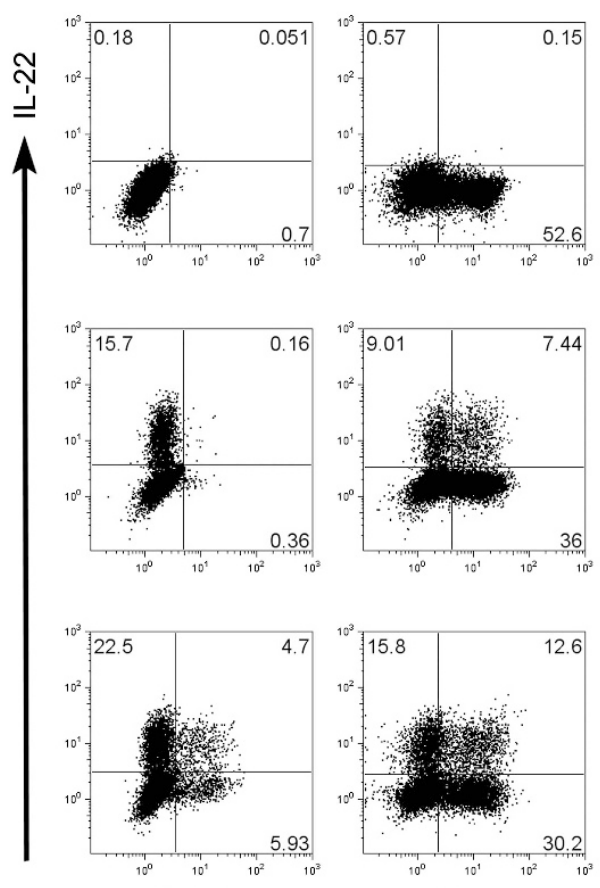

IL-17A

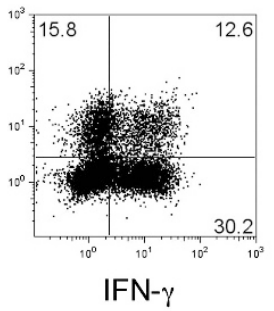

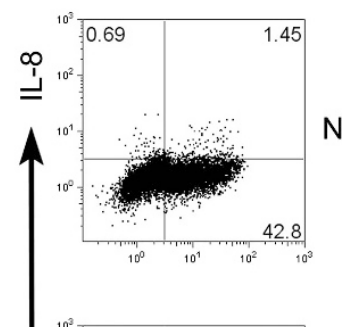

NK

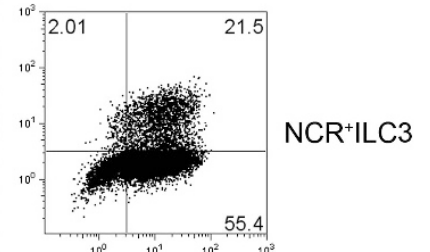

C

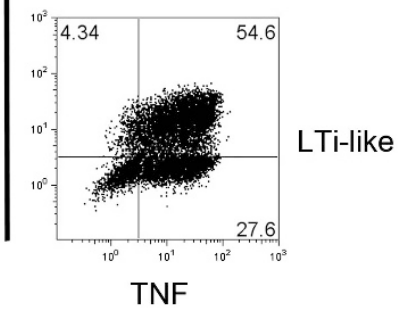

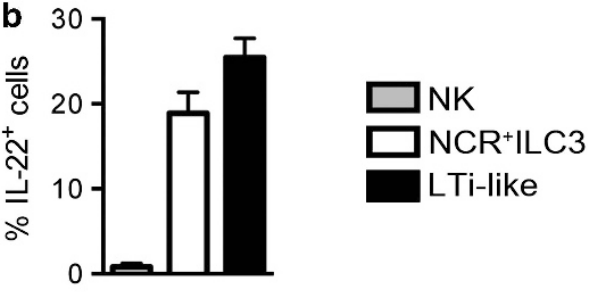
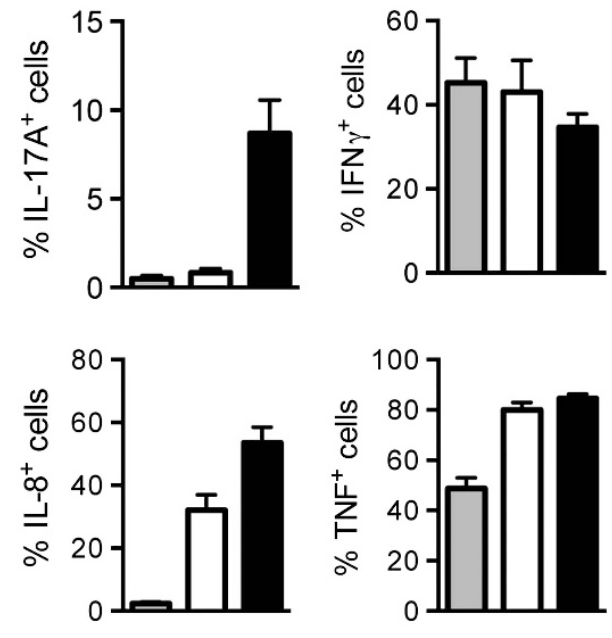

Target K562
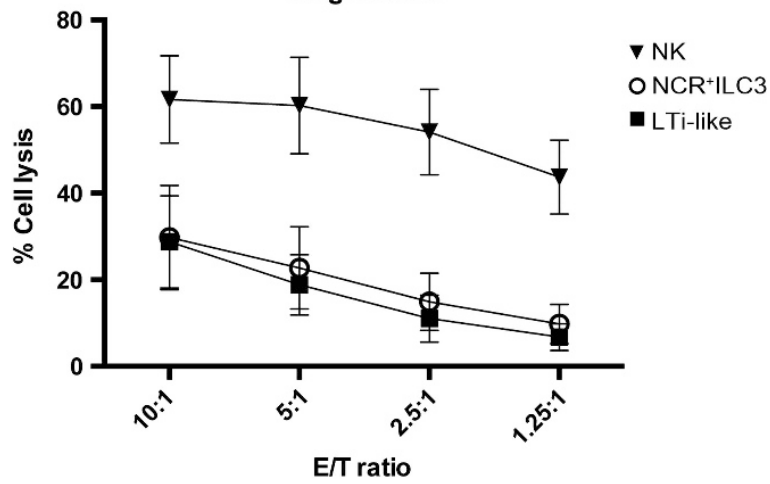

Figure 5 Functional characterization of cultured decidual ILCs. NK cells, NCR ${ }^{+}$ILC3, and LTi-like cells were sorted from decidua and cultured for 20 days in the presence of IL-15. (a, b) Intracellular cytokine analysis of NK cells, NCR ${ }^{+}$ILC3, and LTi-like cells stimulated for $4 \mathrm{~h}$ with PMA/ionomicyn/IL-23. (a) One representative experiment out of seven performed. (b) Mean \pm s.e.m. of percentage of positive cells. (c) Cytolytic activity of NK cells, NCR ${ }^{+}$ILC3, and LTi-like cells was evaluated by ${ }^{51} \mathrm{Cr}$-release assay against $\mathrm{K} 562$ target cells. The effector/target (E/T) ratios are indicated. Mean $\pm \mathrm{s}$.e.m. of percentage of cell lysis obtained in four independent experiments.

the expression of CD94. This suggests that decidual $\mathrm{NCR}^{+}$ ILC3 and LTi-like cells are heterogeneous.

\section{Functional analysis of cultured decidual ILCs}

We next investigated whether cells cultured in IL-15 had modified their cytokine profile. As shown in Figure 5a,b, NK cells, upon PMA/ionomicyn/IL-23 stimulation, maintained the ability to produce primarily IFN- $\gamma$ and TNF and did not produced IL-22, IL-17A, or IL-8. Similar to NK cells, cultured $\mathrm{CD}{ }^{+}{ }^{\mathrm{CD}} 94^{-} \mathrm{CD} 127^{-} \mathrm{CD}_{117}{ }^{-} \mathrm{CD} 103^{+}$ILC1 expressed IFN- $\gamma$ and TNF and mainly maintained their phenotype with the exception of CD94 acquisition (Supplementary
Figure S4a-b). On the other hand, cultured $\mathrm{NCR}^{+}$ILC3 and LTi-like cells enhanced their production of IL-22 and IL-8 as compared with their fresh counterparts, while the production of IL-17A remained restricted to LTi-like cells. In addition, cultured $\mathrm{NCR}^{+}$ILC3 and LTi-like cells also expressed IFN- $\gamma$ and TNF (Figure 5a,b). While $\mathrm{NCR}^{+}$ILC3 maintained their phenotypic and functional characteristics, most LTi-like cells not only acquired CD56 and NKp44, but also a cytokine profile typical of $\mathrm{NCR}^{+}$ILC3. Similar results were obtained in cells cultured with IL-2 (Supplementary Figure S5a-b). In view of the ability of $\mathrm{NCR}^{+}{ }^{+} \mathrm{LC} 3$ and LTilike cells to partially differentiate toward CD94 ${ }^{+}$NK cells, we 
also assessed whether they could mediate cytolytic activity. IL15-cultured $\mathrm{NCR}^{+}$ILC3 and LTi-like cells displayed only marginal cytolytic activity against K562 target cells (Figure 5c). In particular, $\mathrm{NCR}^{+}$ILC3 and LTi-like cells displayed $25 \%$ target cell lysis at an effector/target $(\mathrm{E} / \mathrm{T})$ ratio of 10:1. The same levels of cytolysis was mediated by NK cells and ILC1 at a ratio of $0.3: 1$, which reveals approximately 30 -fold difference in cytolytic activity (Figure 5c and Supplementary Figure S4c). Similar results were obtained with cells cultured in IL-2 (Supplementary Figure S5c). Thus, in line with low perforin and granzyme B expression (Supplementary Figure S2f), both cultured $\mathrm{NCR}^{+}$ILC3 and LTi-like cells are poorly cytolytic. This confirms that these cells could not differentiate toward mature cytolytic NK cells.

\section{A small subset of CD56- ${ }^{-}$LTi-like cells maintain their phenotypic and functional characteristics after culture}

As shown above, cultured LTi-like cells could differentiate toward $\mathrm{NCR}^{+}$ILC3 or even acquire CD94 expression. However, a small fraction (10\%) of LTi-like cells did not acquire the expression of CD56 antigen after culture both with IL-15 or
IL-2 (Figure 6a). After culture, $\mathrm{CD}^{-} 6^{-}$-persisting cell population acquired CD161, NKp44, and partially NKp46 (Figure 6b and Supplementary Figure S6a). Notably, after culture, $\mathrm{CD} 56^{-}$cells maintained high expression of both ROR $\gamma \mathrm{t}$ and CD117, similarly to freshly isolated LTi-like cells (Figure $\mathbf{6 c}$ and Supplementary Figure S6b). In addition, upon PMA/ ionomicyn/IL-23 stimulation, $\mathrm{CD} 56^{-}$cells retained the ability to secrete IL-17A and TNF and also produced IL-8 and IL-22 (Figure 6d and Supplementary Figure S6c). We investigated whether the remaining $\mathrm{CD} 56^{-}$cells maintained their features or rather acquired $\mathrm{NCR}^{+}$ILC3 features upon additional culture. Thus, from LTi-like cells cultured for 20 days, we sorted $\mathrm{CD}^{-} 6^{-}$cells and cultured them for additional 10 or 20 days. As shown in Figure 6e, $\mathrm{CD}^{-} 6^{-}$cells could maintain their $\mathrm{CD} 6^{-} \mathrm{CD} 117^{+}\left(\mathrm{NKp} 44^{-1+}\right)$ surface phenotype after 10 days of culture. However, after 20 days in two out of five experiments, we observed that a fraction of cells became $\mathrm{CD}^{+} 6^{+}$ $\mathrm{CD} 117^{+} \mathrm{NKp} 44^{+}$(not shown). Finally, in limiting dilution cultures of freshly isolated LTi-like cells, plated at three cells per well, we obtained clones that did or did not acquire CD56 (Supplementary Figure S3c,e). Taken together, these data
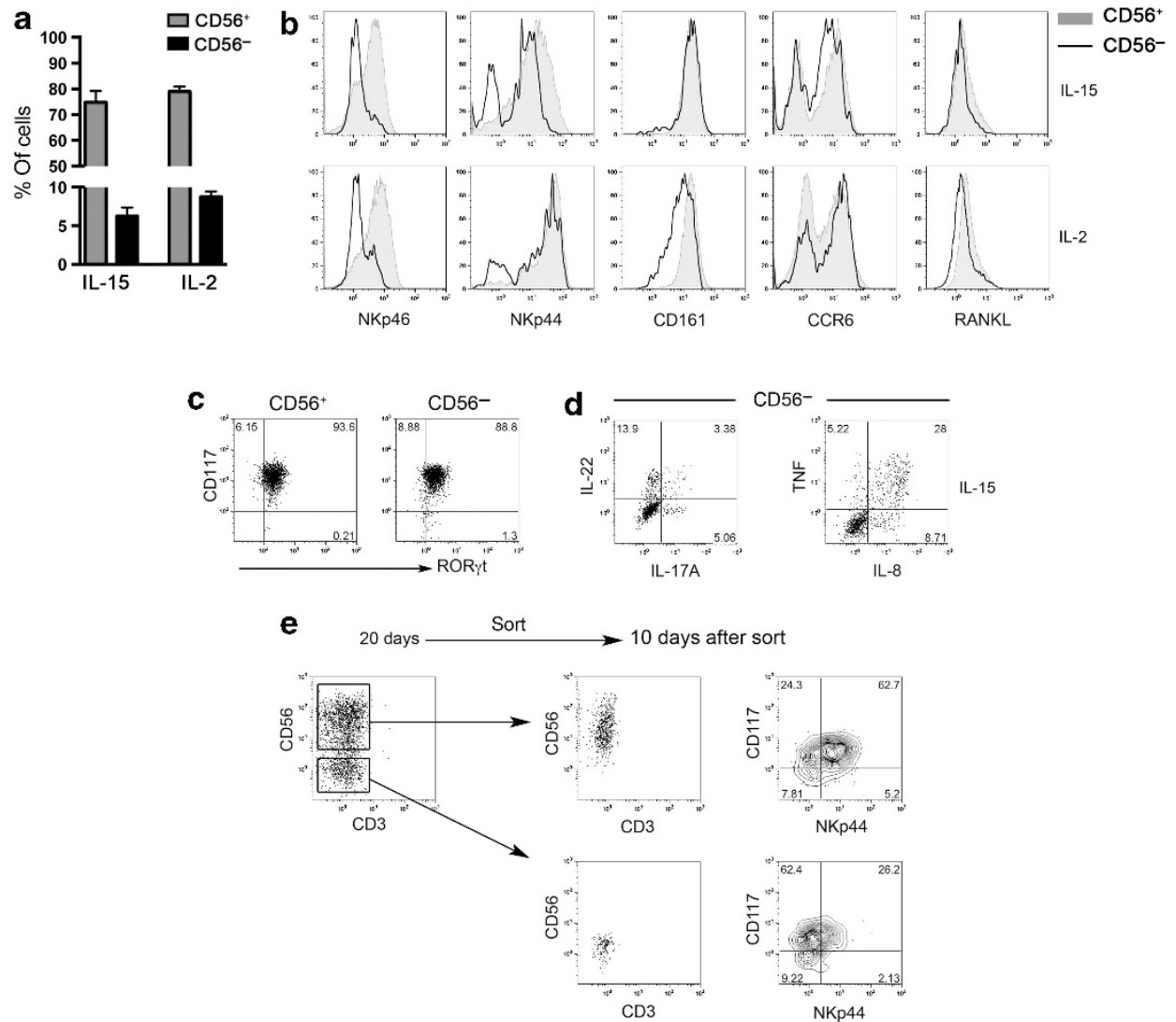

Figure 6 Analysis of cultured CD56 ${ }^{+}$and $\mathrm{CD}_{5}{ }^{-}$decidual LTi-like cells. LTi-like cells were sorted from decidua and cultured for 20 days in the presence of IL-15 or IL-2. (a) Mean \pm s.e.m. of percentages of CD3 ${ }^{-} \mathrm{CD}_{56}{ }^{+}$(gray bars) and CD3 ${ }^{-} \mathrm{CD}^{-} 6^{-}$(black bars) cells. (b) Surface expression of the indicated markers in $\mathrm{CD} 3^{-} \mathrm{CD}_{5} 6^{+}$(filled gray profile) and CD3 ${ }^{-} \mathrm{CD} 56^{-}$cells (black line). One representative experiment out of six performed. (c) Analysis of ROR $\gamma$ t and CD117 expression on $\mathrm{CD}^{-}{ }^{-} \mathrm{CD}_{5}{ }^{+}$and $\mathrm{CD} 3^{-} \mathrm{CD}^{-} 6^{-}$cells cultured in IL-15. One representative experiment out of six performed. (d) Analysis of cytokine production after 4-h stimulation with PMA/ionomicyn/IL-23 by CD3- ${ }^{-}$CD56 $^{-}$cells cultured in IL-15. (e) After 20 days of culture in IL-15, LTi-like cells were sorted again according to CD56 expression and further cultured. After 10 days, cells were analyzed by flow cytometry for the indicated markers. One representative experiment out of three performed. 
suggest that LTi-like cells are heterogeneous, indeed they may contain cells with capability to maintain their phenotypic and functional features as well as to differentiate toward other ILC subsets.

\section{DISCUSSION}

In the present study, we show that different ILC populations are present in human decidua during the first trimester of pregnancy. Indeed, in addition to NK cells, decidua contains other IFN- $\gamma$-producing ILC1. Moreover, we identified two ILC3 populations, $\mathrm{NCR}^{+}$ILC3 and LTi-like cells, characterized by the ability to produce IL-22/IL- 8 and TNF/IL-17A, respectively. Finally, we show that, while $\mathrm{NCR}^{+}$ILC3 substantially maintain their phenotypic characteristics after culture in IL-15 or IL-2, the majority of LTi-like cells acquire the phenotypic and functional features of $\mathrm{NCR}^{+}$ILC3.

Different ILC populations have been recently identified in different tissues, where they play a role in innate responses and contribute to tissue homeostasis and lymphoid tissue generation. ${ }^{2,44}$ The finding that ILC are also present in human decidua, during the first trimester of pregnancy, suggests that these cells may participate in the immune responses necessary for the maintenance/success of pregnancy. The absolute cell number of decidual ILC3 is very small compared with that of NK cells. However, decidual ILC3 have a frequency comparable, if not higher, with that of tonsil ILC3, suggesting that these cells, similarly to tonsil ILC3, ${ }^{21,38,45}$ may play a role in decidual tissues. During early pregnancy, the balance between inflammation and tolerance is critical. Indeed, it has been shown that pro-inflammatory cytokines contribute to tissue remodeling and neo-angiogenesis, thus favoring embryo implantation. The inflammatory phase is followed by a regulatory phase characterized by an increase in Tregs and other regulatory cells that prevent immune-mediated fetal rejection. ${ }^{28}$ Our present results indicate that $\mathrm{NCR}^{+}$ILC3 and LTi-like cells present in decidua are capable of producing pro-inflammatory cytokines including IL-8, IL-22, IL-17A, TNF, and IFN- $\gamma$. These results suggest that ILC3 and ILC1 may be involved in the inflammatory phase of early pregnancy required for embryo implantation. In addition, our data suggest that peculiar functional capabilities, previously thought to be confined to other innate or adaptive immune cells, may be assigned, at least in part, also to ILC populations. For example, it has been proposed that Th17 cells present in the uterus may mediate immune responses against extracellular microbes. ${ }^{28}$ Since decidual ILC produce IL-17A and IL-22, it is conceivable that they contribute to antimicrobial defenses during pregnancy.

For a successful pregnancy, building and remodeling of vessels is a fundamental process, which is favored by the presence of cells releasing pro-angiogenic factors, including IL-8, vascular endolthelial growth factor, and heparin-bindingEGF growth factor. ${ }^{26,27,35,46,47}$ Different studies reported that dNK cells release these soluble factors, in particular IL-8, during the first trimester of pregnancy. However, in light of our present study, also decidual NCR ${ }^{+}$ILC3 appear to contribute to IL-8 production. These data are in line with previous reports showing that tonsil $\mathrm{NCR}^{+}$ILC3 produce IL-8., ${ }^{2,38,39}$ Importantly, after culture in IL-15 or IL-2, decidual $\mathrm{NCR}^{+}$ILC3 and LTi-like cells can upregulate their IL-8 production.

The developmental relationship among different ILC is still a matter of debate. In particular, since the phenotypic features of human-immature NK cells and ILC3 partially overlap, the relationship between NK cells and ILC3 is unclear. ${ }^{21,24,44,48,49}$ It has been proposed that $\mathrm{NCR}^{+}$ILC3 may simply represent an immature stage of NK cell differentiation. Alternatively, they could represent lineage-specified cells. Notably, $\mathrm{NCR}^{+}$ILC3 and LTi-like cells isolated from decidua failed to differentiate into cytolytic mature NK cells, in spite of the partial expression of CD7 (marker of NK cell precursors). Our present results indicate that LTi-like cells are heterogeneous. Indeed, only a small percentage $(10 \%)$ of LTi-like cells retained their phenotypic characteristics $\left(\mathrm{Lin}^{-} \mathrm{CD} 56^{-} \mathrm{CD} 94^{-} \mathrm{CD} 117^{+} \mathrm{ROR}_{\mathrm{t}}{ }^{+}\right.$) upon culture, while the majority of them underwent differentiation toward $\mathrm{NCR}^{+}$ILC3 $\left(\mathrm{Lin}^{-} \mathrm{CD} 56^{+} \mathrm{CD} 94^{-} \mathrm{NCR}^{+/-}{ }^{\mathrm{ROR}} \gamma \mathrm{t}^{+}\right.$ IL-22 ${ }^{+}$). This finding supports the notion of the existence of a developmental relationship between decidual LTi-like cells and $\mathrm{NCR}^{+}$ILC3, as suggested by previous reports in other organs. ${ }^{2,21}$ It cannot be excluded that, similar to NK cells, also $\mathrm{NCR}^{+}$ILC3 and LTi-like cells may derive from a hematopoietic precursor present in decidua. Interestingly, previous studies showed that $\mathrm{dCD} 34$ precursors express ID2 TF, which is required for ILC development. ${ }^{30,36}$ These data suggest that $\mathrm{dCD}^{+} 4^{+}$cells contain not only precursors of dNK cells, but also of NCR ${ }^{+}$ILC3 3 and LTi-like cells. Further studies are required to clarify the origin of decidual ILC3.

In conclusion, our study provides clear evidence that different ILC populations are present in human decidua and also sheds light on the developmental relationship among dNK cells, $\mathrm{NCR}^{+}$ILC3, and LTi-like cells. The ability of ILC1 and ILC3 to release pro-inflammatory cytokines suggests that they may exert an innate antimicrobial role, thus contributing to defenses against infectious agents. In addition, ILC3 may contribute to both tissue building/remodeling and neoangiogenesis through IL-8 production. It is possible that altered ILC differentiation and function may result in pregnancy failure consequent to infection or to deficient tissue and vessel formation.

\section{METHODS}

Isolation and culture of DILs and DSCs. We obtained decidua (d) samples at 9-12 weeks of gestation from singleton pregnancies of mothers requesting termination of pregnancy for social reasons at AOUSM-IST (Genova, Italy). Tonsils were obtained from patients undergoing tonsillectomy at the Giannina Gaslini Institute (Genova, Italy). The relevant institutional review boards approved the study and all patients gave their written informed consent according to the Declaration of Helsinki. We isolated cell suspensions from decidual tissue with GentleMacs (Miltenyi Biotec, Bergisch Gladbach, Germany) and cells were then filtered as previously described. ${ }^{34}$ Decidua-infiltrating lymphocytes were isolated by Fycoll (Cedarlane, Burlington, Ontario) gradient centrifugation. DILs were subsequently analyzed by flow cytometry or stimulated for cytokine production. To obtain single-cell suspension, tonsils were cut in small pieces, minced, and filtered through a $100-\mu \mathrm{m}$ strainer before density gradient 
centrifugation. Tonsil mononuclear cell suspensions were analyzed by flow cytometry. In order to isolate NK cells, $\mathrm{NCR}^{+}$ILC3, or LTi-like cells, DILs were sorted as described in the results section on FACSAria (BD Bioscience, San Jose, CA), purity was routinely $>95 \%$. We plated sorted NK cells, $\mathrm{NCR}^{+}$ILC3, or LTi-like cells at 1,000 cells per well for bulk cultures or using a limiting dilution approach from 200 to 1 cells per well for clonal assays. Cells were cultured in 96-well plates (Corning, Tewksbury, MA) in RPMI-1640 medium (Lonza, Basel, Switzerland) supplemented with $10 \%(\mathrm{vol} / \mathrm{vol})$ fetal calf serum (Lonza), 1\% (vol/vol) glutamine, and 1\% (vol/vol) penicillin, neomycin, and streptomycin antibiotic mixture (Lonza and Cambrex, Charles City, IA), with $50 \mathrm{ng} \mathrm{ml}^{-1}$ IL-7, $50 \mathrm{ng} \mathrm{ml}^{-1} \mathrm{IL}-1 \beta$, (Miltenyi), or 1,000 U ml ${ }^{-1}$ IL-2 (Peprotech, Rocky Hill, NJ), or with feeder cells (irradiated allogeneic peripheral blood mononuclear cells + LCL $221^{\star} \mathrm{G}$ cells) and $10 \mathrm{ng} \mathrm{ml}^{-1}$ IL-15 (Miltenyi) or $100 \mathrm{U} \mathrm{ml}^{-1}$ IL-2. To obtain DSCs, we plated fresh decidual cell suspensions and cultured them in the presence of serum-supplemented RPMI medium, as previously described. ${ }^{50}$ After 7 days, we removed non-adherent cells to separate the adherent cell fraction (DSC). Half the medium volume was replaced twice a week. When the cultures nearly reached confluence, they were harvested by treatment with trypsin/EDTA solution (Lonza) and re-plated at $5 \times 10^{5}$ cells per $75-\mathrm{cm}^{2}$ tissue culture flasks. DSCs were used in the experiments only after 1-2 expansion passages to ensure depletion of monocytes/macrophages. To perform coculture experiments, DSC and ILC were plated at the ratio of $1 / 5$ in the presence of IL-7.

Flow cytometry analyses and monoclonal antibodies. Cells were stained with the following monoclonal antibodies: IL-17A-FITC; IL17A-APC; IL-2-APC; NKp44-biotin; CD335 (NKp46)-APC; CD294 (CRTH2)-PE; CD314 (NKG2D)-PE; CD117-APC; CD3-PE and CD25-PE purchased from Miltenyi, Bergisch Gladbach, Germany; IFN $\gamma$-Alexa647; CD62L-PE; CD45-APC-H7, and CCR6-A647 purchased from BD Bioscience; IL-22-PE; TNF $\alpha$-eFluor450; IFN $\gamma$ PerCP-Cy5.5; ROR $\gamma$ t-PE; CD14-eFluor450; CD19-eFluor450; CD14APC-eFluor480; CD19-APC-eFluor480; ROR $\gamma$ t-APC; Eomes-AlexaFluor647; CD34-PerCP-Cy5.5, and CXCR5-PE purchased from eBioscence, San Diego, CA; CD94-FITC; CD127-BrilliantViolet421; NKp44-APC; CD335-PacifiBlue; CD254 (RANKL)-PE; CD161PerCP-Cy5.5; CD117-PerCP-Cy5.5; CD127-PerCP-Cy5.5; CD7FITC; CD11a(LFA-1)-PerCP and -FITC; CD103-PE purchased from BioLegend, San Diego, CA: IL-8-PE; IL-23R-biotin; IL-1R1-PE and CD335(NKp46)-PE purchased from R\&D, Minneapolis, MN; CD103FITC purchased from ImmonuTools, Friesoythe, Germany; Perforin$\mathrm{PE}$ and CD106 (VCAM1)-PE purchased from Ancell, Bayport, MN CD56-PC7, CD158a,h-PE; CD158e1,e2-PE; CD158b1/b2,j-PE and CD3-ECD purchased from IL-BeckmanCoulter; Streptavidi-AlexFluor700; GranzymeB-PE and Live/dead fixable aqua dead purchased from Invitrogen/Molecular Probes (Carlsbad, CA). ICAM1 was kindly provided by A. Poggi.

For intranuclear staining of TF, cells were stained for surface markers, fixed, and permeabilized with Fixation/Permeabilization buffer (eBioscience) and permeabilization buffer (eBioscience), respectively, according to the manufacturer's instructions. For intracellular cytokine detection, cells were stimulated as indicated in the result section in the presence of $25 \mathrm{ng} \mathrm{ml}^{-1}$ PMA (Sigma), $1 \mu \mathrm{g}$ $\mathrm{ml}^{-1}$ Ionomycin (Sigma-Aldrich, St. Louis, MO), $50 \mathrm{ng} \mathrm{ml}^{-1} \mathrm{IL}-23$, $50 \mathrm{ng} \mathrm{ml}^{-1} \mathrm{IL}-7$, and $50 \mathrm{ng} \mathrm{ml}^{-1}$ IL-1 $\beta$ (Miltenyi) in the presence of GolgiStop (BD). After stimulation, cells were stained for surface markers, fixed, and permeabilized with Cytofix/Cytoperm (BD) and with Perm/Wash (BD), respectively according to the manufacturer's instructions. All samples were analyzed on Gallios Flow Cytometer (Beckman Coulter, Brea, CA). Data analysis was done using FlowJo software (TreeStar, Ashland, OR).

Cytolytic activity. We analyzed cytotoxicity of in vitro (20 days)derived NK cells, NCR ${ }^{+}$ILC3, or LTi-like cells in a 4 -h ${ }^{51} \mathrm{Cr}$-release assay against $\mathrm{K} 562$ target cell line at different effector/target $(\mathrm{E} / \mathrm{T})$ ratios and experiments were performed in duplicates; data are expressed as percentage of target cell lysis.

Statistical analysis. Statistical analysis was performed with Graphpad Prism (La Jolla, CA) software using Friedman test and Dunn's multiple comparison; $\left.\left.P<0.05{ }^{*}\right) ; P<0.01{ }^{* *}\right)$.

SUPPLEMENTARY MATERIAL is linked to the online version of the paper at http://www.nature.com/mi

\section{ACKNOWLEDGMENTS}

We thank CCS directed by Dr A. Bacigalupo, IRCCS-AOUSM-IST, Genoa, Italy for instrumentation provided. This work was supported by AIRC: pr. n. 10225 (L.M.), and n. 9962 (L.M.); MIUR-FIRB 2003 pr. RBLA039LSF-001 (L.M.); Ministero della Salute: RF2006-Ricerca Oncologica-Project of Integrated Program 2006-08, agreements n. RO strategici 3/07 (L.M.) and RO strategici 8/07 (M.C.M.); Progetto Ricerca Ateneo 2013 (P.V.); and ILRC Network project "ImmunoMemory" - Senatsausschuss Wettbewerb (SAW) 2012-15. E.M. is recipient of a fellowship awarded by FIRC.

\section{AUTHOR CONTRIBUTIONS}

P.V. and E.M. designed the study, performed experiments, analyzed data, and wrote the manuscript; D.C. and F.L. performed the experiments; F.C. provided samples and assisted in experiments; P.L.V. provided samples; L.M. and M.C.M. supervised the research and wrote the manuscript.

\section{DISCLOSURE}

The authors declared no conflict of interest.

c 2015 Society for Mucosal Immunology

\section{REFERENCES}

1. Spits, H. et al. Innate lymphoid cells-a proposal for uniform nomenclature. Nat. Rev. Immunol. 13, 145-149 (2013).

2. Spits, H. \& Cupedo, T. Innate lymphoid cells: emerging insights in development, lineage relationships, and function. Ann. Rev. Immunol. 30, 647-675 (2012).

3. Trinchieri, G. Biology of natural killer cells. Adv. Immunol. 47, 187-376 (1989).

4. Caligiuri, M.A. Human natural killer cells. Blood 112, 461-469 (2008).

5. Moretta, A. et al. Activating receptors and coreceptors involved in human natural killer cell-mediated cytolysis. Ann. rev. immunol. 19, 197-223 (2001).

6. Moretta, L. \& Moretta, A. Killer immunoglobulin-like receptors. Curr. Opin. Immunol. 16, 626-633 (2004).

7. Pende, D. et al. Identification and molecular characterization of NKp30, a novel triggering receptor involved in natural cytotoxicity mediated by human natural killer cells. J. Exp. Med. 190, 1505-1516 (1999).

8. Sivori, S. et al. p46, a novel natural killer cell-specific surface molecule that mediates cell activation. J. Exp. Med. 186, 1129-1136 (1997).

9. Vitale, M. et al. NKp44, a novel triggering surface molecule specifically expressed by activated natural killer cells, is involved in non-major histocompatibility complex-restricted tumor cell lysis. J. Exp. Med. 187, 2065-2072 (1998).

10. Kamizono, S. et al. Nfil3/E4bp4 is required for the development and maturation of NK cells in vivo. J. Exp. Med. 206, 2977-2986 (2009).

11. Yokota, Y. et al. Development of peripheral lymphoid organs and natural killer cells depends on the helix-loop-helix inhibitor Id2. Nature 397, 702-706 (1999).

12. Boos, M.D., Yokota, Y., Eberl, G. \& Kee, B.L. Mature natural killer cell and lymphoid tissue-inducing cell development requires Id2-mediated suppression of E protein activity. J. Exp. Med. 204, 1119-1130 (2007).

13. Gascoyne, D.M. et al. The basic leucine zipper transcription factor E4BP4 is essential for natural killer cell development. Nat. Immunol. 10, 1118-1124 (2009). 
14. Aliahmad, P., de la Torre, B. \& Kaye, J. Shared dependence on the DNAbinding factor TOX for the development of lymphoid tissue-inducer cell and NK cell lineages. Nat. Immunol. 11, 945-952 (2010).

15. Gordon, S.M. et al. The transcription factors T-bet and Eomes control key checkpoints of natural killer cell maturation. Immunity 36, 55-67 (2012).

16. Luevano, M., Madrigal, A. \& Saudemont, A. Transcription factors involved in the regulation of natural killer cell development and function: an update. Front. Immunol. 3, 319 (2012).

17. Bernink, J.H. et al. Human type 1 innate lymphoid cells accumulate in inflamed mucosal tissues. Nat. Immunol. 14, 221-229 (2013).

18. Fuchs, A. et al. Intraepithelial type 1 innate lymphoid cells are a unique subset of IL-12- and IL-15-responsive IFN-gamma-producing cells. Immunity 38, 769-781 (2013).

19. Mjosberg, J.M. et al. Human IL-25- and IL-33-responsive type 2 innate lymphoid cells are defined by expression of CRTH2 and CD161. Nat. Immunol. 12, 1055-1062 (2011).

20. Cherrier, M. \& Eberl, G. The development of LTi cells. Curr. Opin. Immunol. 24, 178-183 (2012)

21. Crellin, N.K., Trifari, S., Kaplan, C.D., Cupedo, T. \& Spits, H. Human NKp44 + IL-22 + cells and LTi-like cells constitute a stable RORC + lineage distinct from conventional natural killer cells. J. Exp. Med. 207, 281-290 (2010).

22. Cella, M. et al. A human natural killer cell subset provides an innate source of IL-22 for mucosal immunity. Nature 457, 722-725 (2009).

23. Colonna, M. Interleukin-22-producing natural killer cells and lymphoid tissue inducer-like cells in mucosal immunity. Immunity 31, 15-23 (2009).

24. Cupedo, T. et al. Human fetal lymphoid tissue-inducer cells are interleukin 17-producing precursors to RORC + CD127(+) natural killer-like cells. Nat. Immunol. 10, 66-74 (2009).

25. Luci, C. et al. Influence of the transcription factor RORgammat on the development of NKp46 + cell populations in gut and skin. Nat. Immunol. 10, 75-82 (2009).

26. Hanna, J. et al. Decidual NK cells regulate key developmental processes at the human fetal-maternal interface. Nat. Med. 12, 1065-1074 (2006).

27. Le Bouteiller, P. \& Tabiasco, J. Killers become builders during pregnancy. Nat. Med. 12, 991-992 (2006).

28. Saito, S., Nakashima, A., Shima, T. \& Ito, M. Th1/Th2/Th17 and regulatory T-cell paradigm in pregnancy. Am. J. Reprod. Immunol. 63, 601-610 (2010).

29. Moffett-King, A. Natural killer cells and pregnancy. Nat. Rev. Immunol. 2 , 656-663 (2002).

30. Vacca, P. et al. CD34 + hematopoietic precursors are present in human decidua and differentiate into natural killer cells upon interaction with stromal cells. Proc. Natt. Acad. Sci. USA 108, 2402-2407 (2011).

31. Male, V., Hughes, T., McClory, S., Colucci, F., Caligiuri, M.A. \& Moffett, A. Immature NK cells, capable of producing IL-22, are present in human uterine mucosa. J. Immunol. 185, 3913-3918 (2010).

32. Carlino, C. et al. Recruitment of circulating NK cells through decidual tissues: a possible mechanism controlling NK cell accumulation in the uterus during early pregnancy. Blood 111, 3108-3115 (2008).
33. Kopcow, H.D. et al. Human decidual NK cells form immature activating synapses and are not cytotoxic. Proc. Natl. Acad. Sci. USA 102, 15563-15568 (2005).

34. Vacca, P. et al. Analysis of natural killer cells isolated from human decidua: Evidence that 2B4 (CD244) functions as an inhibitory receptor and blocks NK-cell function. Blood 108, 4078-4085 (2006).

35. Vacca, P. et al. Regulatory role of NKp44, NKp46, DNAM-1 and NKG2D receptors in the interaction between NK cells and trophoblast cells. Evidence for divergent functional profiles of decidual versus peripheral NK cells. Int. Immunol. 20, 1395-1405 (2008).

36. Vacca, P., Moretta, L., Moretta, A. \& Mingari, M.C. Origin, phenotype and function of human natural killer cells in pregnancy. Trends Immunol. 32, 517-523 (2011).

37. Vacca, P. et al. Crosstalk between decidual NK and CD14 + myelomonocytic cells results in induction of Tregs and immunosuppression. Proc. Natl. Acad. Sci. USA 107, 11918-11923 (2010).

38. Glatzer, T. et al. RORgammat $(+)$ innate lymphoid cells acquire a proinflammatory program upon engagement of the activating receptor NKp44. Immunity 38, 1223-1235 (2013).

39. Montaldo, E. et al. Human NK cells at early stages of differentiation produce CXCL8 and express CD161 molecule that functions as an activating receptor. Blood 119, 3987-3996 (2012).

40. Ahn, Y.O., Blazar, B.R., Miller, J.S. \& Verneris, M.R. Lineage relationships of human interleukin-22-producing CD56 + RORgammat + innate lymphoid cells and conventional natural killer cells. Blood 121, 2234-2243 (2013).

41. Cella, M., Otero, K. \& Colonna, M. Expansion of human NK-22 cells with IL7, IL-2, and IL-1beta reveals intrinsic functional plasticity. Proc. Natl. Acad. Sci. USA 107, 10961-10966 (2010).

42. Freud, A.G. et al. Evidence for discrete stages of human natural killer cell differentiation in vivo. J. Exp. Med. 203, 1033-1043 (2006).

43. Freud, A.G., Yu, J. \& Caligiuri, M.A. Human natural killer cell development in secondary lymphoid tissues. Semin. Immunol. 26, 132-137 (2014).

44. Spits, H. \& Di Santo, J.P. The expanding family of innate lymphoid cells: regulators and effectors of immunity and tissue remodeling. Nat. Immunol. 12, 21-27 (2011)

45. Cella, M. et al. A human natural killer cell subset provides an innate source of IL-22 for mucosal immunity. Nature 457, 722-725 (2009).

46. Hanna, J. \& Mandelboim, O. When killers become helpers. Trends Immunol. 28, 201-206 (2007).

47. Jessmon, P., Leach, R.E. \& Armant, D.R. Diverse functions of HBEGF during pregnancy. Mol. Reprod. Dev. 76, 1116-1127 (2009).

48. Hughes, T. et al. Interleukin-1beta selectively expands and sustains interleukin-22 + immature human natural killer cells in secondary lymphoid tissue. Immunity 32, 803-814 (2010).

49. Hughes, T. et al. Stage 3 immature human natural killer cells found in secondary lymphoid tissue constitutively and selectively express the TH 17 cytokine interleukin-22. Blood 113, 4008-4010 (2009).

50. Croxatto, D. et al. Stromal cells from human decidua exert a strong inhibitory effect on NK cell function and dendritic cell differentiation. PloS ONE 9, e89006 (2014) 